\title{
RETRACTION
}

\section{Predicting the site of origin of tumors by a gene expression signature derived from normal tissues}

\author{
E Staub, H-J Buhr and J Gröne
}

Oncogene (2010) 29, 3732; doi:10.1038/onc.2010.184

Retraction to: Oncogene advance online publication, 16 November 2009; doi:10.1038/onc.2009.398

The authors hereby retract the e-publication dated 16 November 2009, entitled, 'Predicting the site of origin of tumors by a gene expression signature derived from normal tissues' and a revised version with the same title was submitted. After the paper published, the authors noticed that an error in the preprocessing of microarray data perturbed the mapping of 145 probeset names to 105 gene symbols that were the basis of the expression signature. The correction required small changes in all figures and tables which made a retraction of the original paper inevitable. After correction of the error the authors state that the qualitative results and the conclusions of the paper remain unchanged. 